\title{
A Review of Traumatic Axonal Injury
}

Dicle Karakaya ${ }^{1}$, [MD]

ORCID: 0000-0003-1939-6802

Ahmet İlkay Işıkay², [MD]

ORCID: 0000-0001-7790-4735

\section{sel ABSTRACT Cen}

Traumatic brain injury is a major cause of mortality and neurological disability worldwide and varies according to its cause, pathogenesis, severity and clinical outcome. This review summarizes a significant aspect of diffuse brain injuries - traumatic axonal injury - important cause of severe disability and vegetative state. Traumatic axonal injury is a type of traumatic brain injury caused by blunt head trauma. It is defined both clinically (immediate and prolonged unconsciousness, characteristically in the absence of space-occupying lesions) and pathologically (widespread and diffuse damage of axons). Following traumatic brain injury, progressive axonal degeneration starts with disruption of axonal transport, axonal swelling, secondary axonal disconnection and Wallerian degeneration, respectively. However, traumatic axonal injury is difficult to define clinically, it should be considered in patients with Glasgow coma score $<8$ for more than six hours after trauma and diffuse tensor imaging and sensitivity-weighted imaging MRI sequences are highly sensitive in its diagnosis. Glasgow coma score at the time of presentation, location and severity of axonal damage are prognostic factors for clinical outcome.

Keywords: Diffuse, traumatic, axonal, injury.

https://doi.org/10.32552/2021.ActaMedica.467

Received: 29 May 2020, Accepted: 9 March 2021

Published online: 8 June 2021

\section{INTRODUCTION}

Traumatic axonal injury (TAI) is a distinct clinicopathological topic that can cause severe neurological disability, vegetative state and mortality. Although this term is used as a separate definition, in 1956, Strich was described as 'diffuse degeneration of white matter' in a group of patients with severe posttraumatic dementia [1]. She showed that shearing injuries at the moment of the rapid acceleration or deceleration trauma, were responsible for the morphological changes in white matter and produced diffuse degeneration of the cerebral white matter [2]. Thereafter, Adams introduced diffuse axonal injury, and graded according to its pathological findings $[3,4]$. In the past few decades, some authors, also as in this review, preferred 'diffuse traumatic axonal injury (dTAI)' or 'traumatic axonal injury (TAI)' instead of diffuse axonal injury (DAI) to describe the etiology of the axonal injury and the pathology because it is not diffuse but actually widespread and/or multifocal [3].

The initial and the basic definition of TAl is a clinicopathological diagnosis that causes severe disability, vegetative state or death after head trauma, in the absence of a space-occupying lesion, without a lucid interval, with sudden and prolonged loss of consciousness. It is pathologically characterized by widespread and disseminated damage of axonal fibers in the brainstem, especially in the pontomesencephalic junction next to the superior cerebellar peduncle, the parasagittal white matter and corpus callosum $[3,4]$.

It is a component of injury in $40-50 \%$ of emergency department admissions due to traumatic brain injury (TBI) and one of the most common cause of severe injury and vegetative condition after closedhead trauma [5]. Moreover, there is evidence 
such as post-traumatic deposition of $A \beta$ and tau proteins, and $A \beta$ plaques and axonal $A \beta$ deposits seen in approximately one-third of patients who died shortly after trauma have shown that this progressive axonal degeneration may play a role in the development of neurodegenerative disorders, including Alzheimer's disease [6,7].

\section{Etiology}

Motor vehicle crashes (MVC) are the major cause of TAI. It is thought that TAI is present as a component of TBI in all traffic accidents in which the patient has lost consciousness. In addition, high-speed collisions of football players or hockey players with rival players can cause TAl. Also, in shaken baby syndrome, the sudden deceleration which is occurred in repetitive shaking on the coronal plane may result in TAI. On the other hand, TAI is rarely seen in simple falls and direct blows to the head $[3,8]$.

\section{Pathophysiology and Biomechanism of TAI}

Two main mechanisms are involved in head injuries: 1) direct contact and 2) acceleration and deceleration. Acceleration forces resulting from sudden movement of the head, initiating the forces of shearing and compressive strains and typically cause two conditions: 1 ) acute subdural hematoma (ASDH) by tearing of the subdural bridging veins and 2) TAl, caused by strain and tearing of the axons due to the gyrencephalic structure of the brain and the high white matter/gray matter ratio.

These two injuries differ in terms of the duration and plane of the acceleration forces the head is exposed to. In experimental studies, it has been shown that ASDH occurs in a relatively short time $(5-10 \mathrm{~ms})$ with high rates of angular acceleration whereas TAl is caused by acceleration force occurring in the coronal plane, lower velocity and longer time (20$25 \mathrm{~ms}$ ) when compared with ASDH $[4,9,10]$.

For this reason, TAl is frequently seen in high-speed motor vehicle accidents, shaken baby syndrome and falls from a considerable height that are exposed to acceleration-deceleration forces lasting more than 1 second in the coronal plane $[3,8]$. It has been also demonstrated that TAl is an important mechanism in cervical spinal cord injury in MVCs in the absence of cervical displacement [11].

\section{Histopathological Features}

Axons in white matter are particularly susceptible to acceleration-deceleration trauma due to their viscoelastic structure, anisotropic arrangement, linear microtubules and neurofilaments, and the axolemma surface being larger than the axoplasm volume [12].

The first consequence of the dynamic deformation of the white matter pathways during trauma is the accumulation of substances, such as amyloid $\beta$ precursor protein (APP) carried along the axons, in axonal swellings with disruption of normal axonal transport (primary axotomy). However, stretch injury without complete axotomy contributes more to the pathology seen in TAI. These axons may appear morphologically normal, but are functionally inadequate [13].

Following the trauma, in addition to physical stretch due to deformation of the brain and direct cytoskleteal damage, disruption of membrane permeability and depolarization also occur. These electrochemical changes trigger the release of excitatory neurotransmitters such as glutamate. As glutamate binds to NMDA and AMPA receptors, calcium entry into the cell increases. Increased cytosolic calcium concentration results in mitochondrial damage. Moreover, further damage occurs in the cytoskeletal structure of the axon by activation of calcium-dependent proteolytic enzymes (calpain and caspases). Oxidative stress and disrupted energy metabolism secondary to mitochondrial damage result in neuronal death. Neuroinflammation and microglial activation also contribute to secondary injury processes. This excitotoxic, oxidative, and inflammatory pathways, which cause progressive axonal deterioration and breakage, is called secondary axotomy $[14,15]$. During this period, axonal retraction balls (axonal bulbs) are formed and the proximal parts of the axons become undulated. Concurrently, Wallerian degeneration begins at the distal of the axons by 24 hours. After 3 days, the damaged and separated segments are surrounded by active microglial cells [5]. Progressive myelin degeneration and atrophy of the white matter occur within the first 2 years after TAl and represents the final stage of the process [8]. In the study of Mu et al., apoptosis of oligodendrocytes in the corpus callosum and brainstem shows oligodendrocyte selective injury in TAI. Axonal 
degeneration secondary to demyelination in the brainstem is another pathological pattern of TAI [16]. Briefly, posttraumatic axonal degeneration develops as disruption of axonal transport, axonal edema secondary to disconnection, and Wallerian degeneration, respectively [17].

Pathologically, axonal retraction balls, axonal swelling (axonal varicosity) and punctate hemorrhages (Strich hemorrhages) are observed in corpus callosum, internal capsule, cerebral white matter, around the third ventricule, fornix, brainstem and cerebellum. The presence of retraction balls is important for showing complete axonal disruption [7].

The histopathological diagnosis of TAl depends on demonstrating the abnormal axonal profiles as described above. Since the hematoxylin-eosin and various silver impregnation techniques are insufficient to demonstrate the entire axonal injury in the damaged tissue, its usefulness in defining the structural features is limited. Therefore, immunohistochemical staining of APP, which is transferred by fast axonal transport and may be identified in damaged axons within 2 hours after trauma, is still the gold standard for the clinicopathological diagnosis. However, APP accumulation is not specific for traumatic brain injury, it should be noted that it can also be seen in hypoxic/ischemic injury [13]. The detection of single or small groups of diffuse, messy, 'sausagelike' or 'varicosity-like', $\beta$-APP-positive swollen axons or retraction bulbs, surrounded by a clean background can be considered as traumatic origin of axonal damage. In contrast, circumscribed foci or a linear pattern described as 'zigzag' or 'Z-shaped' pattern of $\beta$-APP-positive axons that are found in a dirty, granular background is seen in hypoxic/ ischemic axonal damage [4].

In the pathological determination of TAl, the lesions described above must be located at least one is above and one is below the tentorium, and especially on the axonal tracts such as corpus callosum and internal capsule, with a typical traumatic appearance, pattern and distribution [4]. The Adams classification is used for grading of TAI as mild, moderate or severe. In this classification, mild (grade 1) is characterized by microscopic axonal changes in the white matter of the cerebral hemispheres including the corpus callosum, brainstem and occasionally the cerebellum. In moderate (grade 2) TAI, in addition to grade 1, there is a focal lesion in the corpus callosum. And lastly, severe (grade 3) TAI is defined since there are additional focal lesions in the dorsolateral brainstem, commonly in the superior cerebellar peduncle [18]. It is obvious that primary brainstem lesions are usually associated with TAl and includes cerebral hemispheres and cerebellum as well as brainstem [8].

\section{Clinical Diagnosis and Evaluation}

TAl is a clinical diagnosis and often 'diagnosis of exclusion'. It is thought that TAI has occurred whenever there is any loss of consciousness. Clinical presentation is related to its severity. For instance, in mild TAl, symptoms and signs are similar to concussive disorder, such as headache, dizziness, nausea, vomiting, and weakness. But severe TAI may present with loss of consciousness, severe neurological deficits, persistent vegetative state and death. Severity and duration of coma is related to the injury of the reticular activating system.

In clinical setting, the diagnosis is made by clinical information and radiologic findings. It is important to understand the mechanism of head trauma in order to make the differential diagnosis of TAI. Notably, it should be considered in patients with Glasgow coma score (GCS) $<8$ for more than six hours after trauma.

Computed tomography (CT) is the fastest and easily accessible option for evaluating TBI, especially for focal injuries and intracranial hematomas that may need immediate surgery. Nevertheless, initial CT scans after trauma are frequently normal in TAI. Only $10 \%$ of patients have classical CT findings include small, punctate hemorrhages in corpus callosum, white matter of the cerbral hemispheres and brainstem (Figure 1. A-C). Enlargement of the ventricles may also be seen in CT within 2 weeks after trauma. Late $\mathrm{CT}$ findings may be relatively normal, except for atrophy of the parasagittal white matter with ventricular enlargement [8].

Magnetic resonance imaging (MRI) is superior to $C T$ in showing TAl lesions. Diffuse tensor imaging (DTI) and diffusion weighted imaging (DWI) are the preferred imaging methods for the diagnosis of TAI. In gradient-echo sequences, microhemorrhages which are seen as hypointense foci can be detected with high sensitivity. The prevalence of microhemorrhages in gradient- 

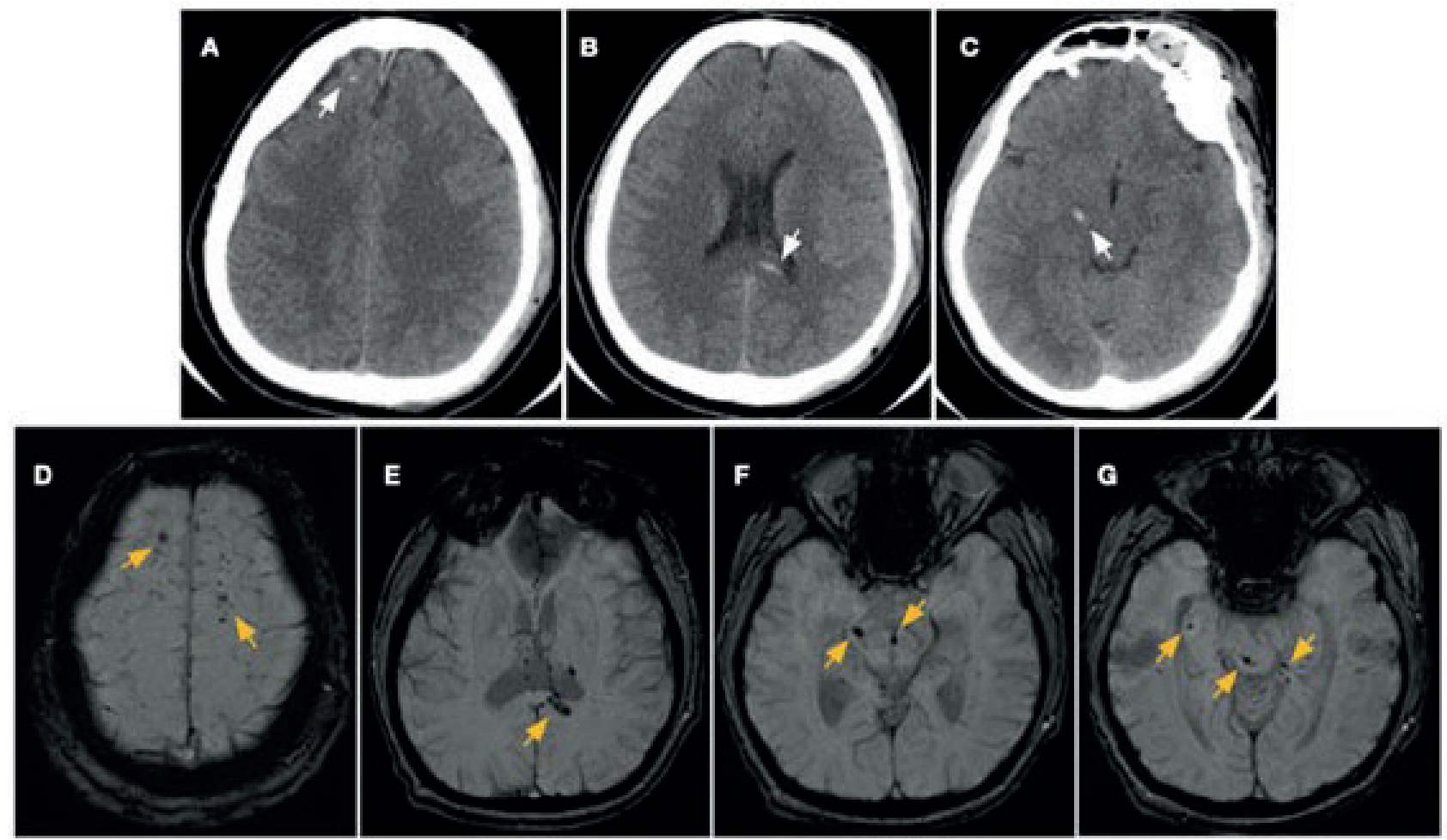

Figure 1. CT and SWI MRI scans. A-C) Petechial hemorrhages in the right frontal white matter, splenium of the corpus callosum and right thalamus on CT imaging (white arrow). D-G) Hypointense microhemorrhage foci (yellow arrow) along the body and splenium of the corpus callosum, right cerebral peduncle, mesencephalon and bilateral mesial temporal white matter in SWI (Courtesy of Melike Mut Aşkun, MD, PhD).

echo images correlates well with the patient's GCS [13]. In addition, susceptibility weighted imaging (SWI), which is a modification of the gradientecho sequence, is more sensitive in demonstrating petechial hemorrhages (Figure 1. D-G) [19].

Currently, there is no ideal laboratory test for diagnosing and indicating the prognosis of TAl. However, there are up-to-date researches to identify the biomarkers such as neurofilaments, myelin basic protein, tau protein, amyloid protein, and spectrin degradation products in body fluids (serum, cerebrospinal or brain extracellular fluid) at different time points after traumatic brain injury, which can improve immediate triage of patients and yield prognostic information $[20,21]$.

\section{Treatment}

Implementation of the basic life support protocol is a standard approach for all patients suffering from TBI. Treatment of TAl is aimed at preventing of secondary damage and facilitating rehabilitation. Therefore, hypotension, hypoxia, cerebral edema and elevated intracranial pressure should be avoided to save some axons by preventing or reducing the effects of secondary cascades that cause secondary axotomy.
In the last decade, with the understanding of TAI pathophysiology, significant progress has been made to better targeting intracellular mechanisms that may be most suitable for therapeutic intervention. It has been found that in vitro stabilization of microtubules with taxol inhibits secondary depolymerization of microtubules, thereby reducing swelling and degeneration. Although it has not yet been tested in animal models, the fact that taxol-like therapy (taxanes), recently aimed at the treatment of Alzheimer's and other neurodegenerative diseases, may open the way for this therapeutic approach for TAI [22].

In another in vitro model, it has been shown that increases in intraaxonal calcium concentrations are inhibited by calcineurin, calcium-activated phosphatase, demonstrating intracellular release of calcium after trauma. However, further studies are needed to determine its effectiveness in vivo. A few in vivo studies have also examined various 'axonoprotective' treatments that target calciummediated pathways, directly or indirectly. After TBI, activation of calpain, a calcium-dependent cystein protease which is responsible for degredation of subaxolemmal spectrin and impairment of axonal cytoskeleton, is inhibited by calpain inhibitors such 
as MDL28170, 5b, AK295, and SJA-6017. But their protective effects are controversial [22].

For targeting mitochondrial damage and dysfunction, Okonkwo et al. prevented the opening of the transition pores that caused mitochondrial permeability by immunophilin ligand cyclosporin A $[23,24]$. Treatments aimed to membrane fluidity and repair, such as polyethylene glycol and surfactant, may be beneficial for pores in axolemma [25]. Several studies also promote the use of free radical scavengers to prevent secondary axotomy. Eventually, newly identified protective agents should undergo more rigorous preclinical evaluation to better position these agents for use in human clinical trials.

Since TAI is a process that occurs with progressive axonal degeneration and neuronal cell death, stem cells which are renewable, show region-specific differentiation and migrate to targets, can be used in the treatment of TAI and rehabilitation of the patient, but still need to be developed and a promising treatment choice [26].

\section{Prognosis}

Comparison of patients with and without TAI has shown that patients with TAl have an almost three times higher risk of unfavourable outcome [27]. Mean GCS at the presentation, rather than its degree, is a better predictor of clinical outcome [28]. GCS reflects the amount of midbrain and brainstem injury more accurately than cortical damage. Therefore, it is a more accurate determinant than other traumatic brain injury measures such as the amount of cortical contusion seen on CT [8].

Functional recovery is determined by the location and severity of axonal injury. It has been demonstrated that the probability of neurological recovery is decreased when there are many large lesions located in the pons and midbrain. Anticipating the prognosis of grade 3 TAl, it would be appropriate to consider the number, size and location of lesions in the brainstem $[29,30]$. However, presence of lesions in dorsal brainstem, especially the ascending reticular activating system (ARAS), has more prognostic significance than the total number of lesions in the whole brain or brainstem [31].

\section{Complications}

Injury of the tracts entering and leaving the hypothalamus and/or direct injury to the pituitary stalk and gland, causes common medical complications, including dysautonomia, hormonal disturbances, salt and water imbalance, and altered temperature regulation.

Over the years, dysautonomia associated with TAl has been named in different ways, but today, paroxysmal sympathetic hyperactivity (PSH) is accepted as the most accurate term. The definition of PSH is 'a syndrome, recognized in a subgroup of survivors of severe acquired brain injury with simultaneous, paroxysmal, transient increases in sympathetic (elevated heart rate, blood pressure, respiratory rate, temperature, sweating) and motor (posture) activity' [32]. Typically, symptoms of autonomic dysregulation such as tachycardia, tachypnea, hypertension, hyperthermia, diaphoresis, decerebrate / decorticate posture, dystonia, rigidity / spasticity are observed. It is a life-threatening condition seen in $8-33 \%$ of adults and $13 \%$ of pediatric patients after severe traumatic brain injury $[33,34]$. Even afferent stimuli such as endotracheal aspiration, bed positioning, bathing and muscle stretching or passive exercises are potentially nociceptive in patients with dysotonomia and may provoke PSH [35].

Diagnosis of PSH is made by excluding undiagnosed fractures, heterotopic ossification, painful spasticity / dystonia, withdrawal of opiates or sedatives, epileptic seizures, neuroleptic malignant syndrome, malignant hyperthermia and sepsis. Treatment with dopaminergic agents (bromocriptine), a-2 adrenergic agonists (clonidine, dexmedetomidine), opiates, $\beta$-adrenergic antagonists (propranolol) and benzodiazepines may reduce the symptoms in patients with PSH. In addition, local botulinum toxin A or phenol injection can be applied.

\section{CONCLUSION}

Overall, TAI is a complex process that begins with stretching and shearing of the axons, sometimes with a permanent disconnection and resulting in long-lasting functional impairment.

In our clinic, approximately 900 patients were followed up with a diagnosis of TBI between 2010 
and 2020. 32 patients were diagnosed with TAl, and 29 of these patients presented with moderate to severe head trauma. Mortality rate is $12.5 \%$ (4/32). Although there is improvement in understanding of its pathophysiology, biomarkers and potential therapeutic targets, still the main purpose of the treatment is supportive care and prevention of secondary injuries.

\section{CONFLICT Of INTEREST STATEMENT}

The authors declare they have no conflicts of interest.
[1] Strich SJ. Diffuse Degeneration of the Cerebral White Matter in Severe Dementia Following Head Injury. J Neurol Neurosur Ps. 1956; 19(3): 163-185.

[2] Strich SJ. Shearing of Nerve Fibres as a Cause of Brain Damage Due to Head Injury - a Pathological Study of 20 Cases. Lancet. 1961; 2(720): 443-448.

[3] Davceva N, Sivevski A, Basheska N. Traumatic axonal injury, a clinical-pathological correlation. J Forensic Leg Med. 2017; 48: 35-40.

[4] Davceva N, Basheska N, Balazic J. Diffuse Axonal Injury-A Distinct Clinicopathological Entity in Closed Head Injuries. Am J Forensic Med Pathol. 2015; 36(3): 127-133.

[5] Su E, Bell M. Diffuse Axonal Injury. Front Neurosci. 2015; 57: 41-83.

[6] Fleminger S, Oliver DL, Lovestone $S$, et al. Head injury as a risk factor for Alzheimer's disease: the evidence 10 years on; a partial replication. J Neurol Neurosurg Psychiatry. 2003; 74(7): 857-862.

[7] McKee AC, Daneshvar DH. The neuropathology of traumatic brain injury. In: Traumatic Brain Injury, Part I. 2015: 45-66.

[8] Meythaler JM, Peduzzi JD, Eleftheriou E, et al. Current concepts: Diffuse axonal injury-associated traumatic brain injury. Archives of Physical Medicine and Rehabilitation. 2001; 82(10): 1461-1471.

[9] Zhang JY, Yoganandan N, Pintar FA, et al. Role of translational and rotational accelerations on brain strain in lateral head impact. Biomed Sci Instrum. 2006; 42: 501506.

[10] Li JR, Zhang JY, Yoganandan N, et al. Rotational acceleration duration affects brain strains in lateral impact. Proceeding of the Asme Summer Bioengineering Conference - 2007. 2007: 913-914.

[11] Kakulas BA. The applied neuropathology of human spinal cord injury - In reply to Professor Dietz. Spinal Cord. 1999; 37(8): 595.

[12] Smith DH, Meaney DF, Shull WH. Diffuse axonal injury in head trauma. J Head Trauma Rehab. 2003; 18(4): 307-316.

[13] Johnson VE, Stewart W, Smith DH. Axonal pathology in traumatic brain injury. Exp Neurol. 2013; 246: 35-43.

[14] Lin Y, Wen L. Inflammatory response following diffuse axonal injury. Int J Med Sci. 2013; 10(5): 515-521.
[15] Frati A, Cerretani D, Fiaschi Al, et al. Diffuse Axonal Injury and Oxidative Stress: A Comprehensive Review. Int J Mol Sci. 2017; 18(12).

[16] Mu J, Li M, Wang T, et al. Myelin Damage in Diffuse Axonal Injury. Frontiers in Neuroscience. 2019;13: 217.

[17] Hill CS, Coleman MP, Menon DK. Traumatic Axonal Injury: Mechanisms and Translational Opportunities. Trends Neurosci. 2016; 39(5): 311-324.

[18] Adams JH, Doyle D, Ford I, et al. Diffuse axonal injury in head injury: definition, diagnosis and grading. Histopathology. 1989; 15(1): 49-59.

[19] Marquez de la Plata C, Ardelean A, Koovakkattu D, et al. Magnetic resonance imaging of diffuse axonal injury: quantitative assessment of white matter lesion volume. J Neurotrauma. 2007; 24(4): 591-598.

[20] Manivannan S, Makwana M, Ahmed Al, et al. Profiling biomarkers of traumatic axonal injury: From mouse to man. Clinical Neurology and Neurosurgery. 2018; 171: 6-20.

[21] Ma J, Zhang K, Wang Z, et al. Progress of Research on Diffuse Axonal Injury after Traumatic Brain Injury. Neural Plasticity. 2016; 2016: 1-7.

[22] Smith DH, Hicks R, Povlishock JT. Therapy Development for Diffuse Axonal Injury. J Neurotraum. 2013; 30(5): 307-323.

[23] Okonkwo DO, Buki A, Siman R, et al. Cyclosporin A limits calcium-induced axonal damage following traumatic brain injury. Neuroreport. 1999; 10(2): 353-358.

[24] Okonkwo DO, Povlishock JT. An intrathecal bolus of cyclosporin A before injury preserves mitochondrial integrity and attenuates axonal disruption in traumatic brain injury. J Cereb Blood Flow Metab. 1999; 19(4): 443451.

[25] Serbest G, Horwitz J, Jost M, et al. Mechanisms of cell death and neuroprotection by poloxamer 188 after mechanical trauma. Faseb J. 2006; 20(2): 308-310.

[26] Li XY, Feng DF. Diffuse axonal injury: Novel insights into detection and treatment. J Clin Neurosci. 2009; 16(5): 614619.

[27] van Eijck MM, Schoonman GG, van der Naalt J, et al. Diffuse axonal injury after traumatic brain injury is a prognostic factor for functional outcome: a systematic review and meta-analysis. Brain Inj. 2018; 32(4): 395-402. 
[28] Lohani S, Bhandari S, Ranabhat K, et al. Does Diffuse Axonal Injury MRI Grade Really Correlate with Functional Outcome? World Neurosurg. 2020; 135: e424-e426.

[29] Sandhu S, Soule E, Fiester P, et al. Brainstem Diffuse Axonal Injury and Consciousness. J Clin Imaging Sci. 2019; 9: 32.

[30] Humble SS, Wilson LD, Wang L, et al. Prognosis of diffuse axonal injury with traumatic brain injury. J Trauma Acute Care Surg. 2018; 85(1): 155-159.

[31] Izzy S, Mazwi NL, Martinez S, et al. Revisiting Grade 3 Diffuse Axonal Injury: Not All Brainstem Microbleeds are Prognostically Equal. Neurocrit Care. 2017; 27(2): 199-207.

[32] Baguley IJ, Perkes IE, Fernandez-Ortega JF, et al. Paroxysmal sympathetic hyperactivity after acquired brain injury: consensus on conceptual definition, nomenclature, and diagnostic criteria. J Neurotrauma. 2014; 31(17): 15151520.
[33] Branstetter JW, Ohman KL, Johnson DW, et al. Management of Paroxysmal Sympathetic Hyperactivity with Dexmedetomidine and Propranolol Following Traumatic Brain Injury in a Pediatric Patient. J Pediatr Intensive Care. 2020; 9(1): 64-69.

[34] Lv LQ, Hou LJ, Yu MK, et al. Risk factors related to dysautonomia after severe traumatic brain injury. J Trauma. 2011; 71(3): 538-542.

[35] Baguley IJ, Heriseanu RE, Nott MT, et al. Dysautonomia after severe traumatic brain injury: evidence of persisting overresponsiveness to afferent stimuli. Am J Phys Med Rehabil. 2009; 88(8): 615-622. 